\title{
Utilization of Traditional Anticoagulation for Venous Thromboembolism in Patients with Liver Cirrhosis: Is It Safe?
}

\author{
Saad Qadwai ${ }^{1 *}$, Tayyaba Rehman ${ }^{2}$ and Zeeshan Solangi ${ }^{3}$ \\ ${ }^{1}$ Department of Internal Medicine, Institute of Gaylord Hospital, USA \\ ${ }^{2}$ Department of MITG, Medical Affairs, Institute of Medtronic, USA \\ ${ }^{3}$ Department of Internal Medicine, Institute of Geisinger Medical Center, Danville
}

Received: 阱April 30, 2018; Published: 制 May 08, 2018

*Corresponding author: Saad Qadwai, Department of Internal Medicine, Institute of Gaylord Hospital, 50 Gaylord Farm Road, Wallingford, CT 06492, USA

\begin{abstract}
Patients with liver cirrhosis are known to have increased risk bleeding particularly from gastrointestinal tract. However, recent literature has shown that patients with liver cirrhosis are also at increased risk of developing deep venous thrombosis and pulmonary embolism. Therefore, it is important to consider prophylactic and therapeutic anticoagulation in cirrhotic patients. In this article, we have reviewed the available literature on the safety and efficacy of the utilization of prophylactic and therapeutic anticoagulation in cirrhotic patients.
\end{abstract}

Abbreviations: INR: International Normalized Ratio; VTE: Venous Thromboembolism; DVT: Deep Venous Thrombosis; PE: Pulmonary Embolism; PTT: Partial Thromboplastin Time; LMWH: Low-Molecular-Weight Heparin; UFH: Unfractionated Heparin; CLD: Chronic Liver Disease

\section{Introduction}

Liver plays central physiologic role in hemostasis as it synthesizes the majority of the procoagulant and anticoagulant factors. The levels of these factors are markedly affected by decrease function of liver associated with cirrhosis resulting in abnormal hemostatic mechanism. Generally, the impression in the clinical world is that liver cirrhosis is associated with decrease synthesis of procoagulant factors resulting in increased risk of bleeding. This phenomenon is known as auto-anticoagulation and is supported by elevated international normalized ratio (INR) and low platelet count usually observed in cirrhotic patients. In this regard, gastrointestinal bleeding and more specifically variceal bleed are of major concern since they contribute significantly to the mortality of patients with liver cirrhosis. At least, 30\% mortality has been reported at the first episode with a 70\% recurrence rate in this patient population and a 1year survival estimate ranging from $32 \%$ to $80 \%$ [1]. However, decrease function of cirrhotic liver also results in reduce level of anticoagulant factors including antithrombin III, protein S, and C which may result in increased tendency to form clots. Interestingly, recent data has also noted cases of venous thromboembolism (VTE) including both deep venous thrombosis (DVT) and pulmonary embolism (PE) in cirrhotic patients ranging between $0.5 \%$ to $6.3 \%$ [2-10]. Dabbagh et al. [4] found that even an elevated INR $>2.2$ was not protective against VTE in this patient population. Gulley D et al. [10] noted that hospitalized cirrhotic patients without predisposing co-morbidities (e.g. neoplasm, congestive heart disease and chronic renal failure) had similar risks for VTE as compared to noncirrhotic patients [10]. Thus, the myth of auto-anticoagulation seems to be only partially true. Therefore, the abnormal routine blood tests (like elevated INR, Partial Thromboplastin Time [PTT], high MELD score and low platelet count) may indicate increased hemorrhage risk in this patient population but may not be necessarily completely true as these tests do not accurately reflect the activity of aforementioned anticoagulant factors in the serum. The purpose of this article is to review the epidemiological data available on the utilization of traditional VTE prophylaxis and treatment on the increased bleeding risk in patients with liver cirrhosis.

\section{Methods}

An electronic Medline search was conducted using the key terms anticoagulation, low-molecular-weight heparin (LMWH), 
unfractionated heparin (UFH), oral anticoagulant, deep venous thrombosis, pulmonary embolism, venous thromboembolism, liver cirrhosis, chronic liver disease, and decompensated liver disease. Studies written in the English from January 2000 to March 2018 were considered for this review article. All search results were reviewed.

\section{Results}

Intagliata $\mathrm{N}$ et al. [11] studied the complication rates associated with administration of thromboprophylaxis in 235 hospitalized cirrhosis patients admitted to the University of Virginia between 2007 and 2010 accounting for 355 discrete hospitalizations. They noticed only nine gastrointestinal bleeding events $(2.5 \%$ of admissions) and concluded that VTE prophylaxis was not associated with increased risk of bleeding or death in their patient population [11]. Vivarelli $M$ et al. [12] conducted a retrospective study in Italy on 229 consecutive cirrhotic patients with HCC who underwent hepatic resection. They assess the safety and effectiveness of prophylaxis for VTE in terms of thrombotic or hemorrhagic complications between those who received and those who did not receive prophylaxis with low-molecular weight heparin. They divided the patients into two groups i.e. Group A included $68.5 \%$ of patients and Group B included $31.5 \%$ patients. Group B patients had higher Child-Pugh and MELD scores, lower platelet counts, a higher prevalence of esophageal varices and higher requirements for intraoperative transfusion of FFP. Group A received VTE prophylaxis with LMWH while Group B did not receive VTE prophylaxis. The cases of VTE were higher in group B (1.38\%) as compared to the group A $(0.63 \%)$ but the difference was not statistically significant $(P=0.530)$. On the other hand, the prevalence of hemorrhagic complications was higher in group A as compared to group B but again the difference was not statistically significant $(\mathrm{P}=0.380)$. Moreover, only the presence of varices was associated with increased risk of bleeding $(\mathrm{P}=0.05)$ among other studied risk factors including age, CTP class and Model for End-stage Liver disease scores, platelet count and intraoperative transfusion requirements [12].

Bechmann L et al. [13] conducted a retrospective study in Germany to evaluate the safety of LMWH utilization for either prophylactic or therapeutic indications anticoagulation among 84 patients with cirrhosis. Only seven patients developed variceal bleeding at a rate comparable with the baseline rate in patients with advanced cirrhosis. The hemorrhagic complications were either secondary to bleeding esophageal varices or 2 whypertensive gastropathy with no mortality. They concluded that a prophylactic use of LMWH in patients with cirrhosis appears to be safe [13].

Smith C et al. [14] also conducted a retrospective chart review of 410 chronic liver disease (CLD) patients admitted to a tertiary care academic medical center in US from August 2009 through July 2011. 225 (55\%) patients received thromboprophylaxis including mechanical (154), pharmacologic (49), and combined (22). They noticed a significant decrease in overall thromboprophylaxis use and pharmacologic prophylaxis use for patients with INR $>2.0$ as compared to those with INR 1.4 to $2.0(\mathrm{P}=0.013$ and $\mathrm{P}<0.001$, respectively). $0.7 \%$ of patients developed VTE (DVT) and fifteen (3.7\%) patients had bleeding event. Out of fifteen patients who developed bleeding, nine were on mechanical prophylaxis, 1 on pharmacologic, 3 on combination, and 2 with no prophylaxis. Majority of patients experiencing a bleeding event had an INR $>2.0(\mathrm{P}=0.001)$. They concluded that use of VTE pharmacologic prophylaxis does not appear to increase bleeding risk in CLD patients with INR $\leq 2.0$ [14].

Barclay S et al. [15] conducted another retrospective study to determine whether pharmacologic prophylaxis for VTE was associated with a decrease in the incidence of VTE or an increased incidence of bleeding in patients with CLD. The study sample consisted of a total of 1581 CLD patients hospitalized over a 3-year period and divided into two groups based on receipt of pharmacologic VTE prophylaxis. 392 (24.7\%) patients received pharmacologic VTE prophylaxis. Decrease risk of VTE $(0.5 \%$ vs $1.8 \%, \mathrm{p}=0.05)$ and bleeding ( $2.0 \%$ vs $10.3 \%, \mathrm{p}<0.001)$ were noted in the prophylaxis group as compared to the nonprophylaxis group [15].

In a multicenter retrospective study conducted in 5 hospitals, Reichert J et al, studied the pharmacologic VTE prophylaxis on risk of hemorrhage while hospitalized in patients with CLD and concurrent coagulopathy. They utilized the ICD-9 codes to identify subjects with CLD admitted from January 1, 2012, until December 31, 2012. 256 patients met criteria for analysis, with 80 received pharmacologic VTE prophylaxis and 176 did not receive pharmacologic VTE prophylaxis. They found that pharmacologic thromboprophylaxis was associated with increased number of hemorrhagic events among cirrhotic patients who received it as compared to those who did not $(17.5 \%$ vs $7.4 \%, \mathrm{p}=0.02)$. However, it was noted that difference in rate of overall hemorrhage was driven primarily by a difference in minor hemorrhage [16].

\section{Conclusion}

The use of anticoagulation for VTE prophylaxis and treatment in cirrhosis remains underutilized and it is still not a universal practice to provide hospitalized cirrhotic patients with such prophylaxis and treatment due to the concerns of increased bleeding risk. Also, the literature on the safety and efficacy of anticoagulation in liver cirrhosis is in initial stages and is based mainly on retrospective studies. Six studies have investigated the use of VTE prophylaxis. Two studies have shown that DVT prophylaxis does decrease the risk VTE in cirrhotic patients $[12,15]$. However, the results of one of the study did not reached the statistical significance which could be due to low power of the study because of the small sample size [12]. On the other hand, only one study have shown that pharmacologic thromboprophylaxis was associated with increased number of hemorrhagic events among cirrhotic patients which they attributed 
to increased incidence of minor hemorrhage [16]. Another study noticed higher prevalence of hemorrhagic complication in VTE prophylaxis group as compared to cirrhotic patients who did not received VTE prophylaxis, but their result did not achieve statistical significance [12]. Moreover, only the presence of varices was associated with increased risk of bleeding.

It is possible that risk of hemorrhage is underestimated in these studies because of retrospective nature of studies with possibility of underreporting of bleeding events and underutilization of thromboprophylaxis of VTE in patients at higher risk of bleeding such as those with high INR, low platelets and presence of esophageal varices. Only one study investigated the use of VTE treatment in cirrhotic patients and they found no increased bleeding risk with it [13]. However, it was a small sampled study and there was no control group utilized to compare [13]. In general, recent data suggest that utilization of VTE prophylaxis in cirrhotic patients is safe and should be considered. Underutilization of both chemical and mechanical VTE prophylaxis in hospitalized cirrhotic patients has been noted ranging to as high as $75 \%[8,17]$. In another study, only $9 \%$ of hospitalized patients with CLD were treated with pharmacological VTE prophylaxis, while only 16\% received mechanical VTE prophylaxis [2]. The major concern regarding the use of anticoagulation in this population is the gastrointestinal bleed and specifically esophageal variceal bleed. However, VTE prophylaxis appears relatively safe in cirrhotic patients and should be considered in all hospitalized cirrhotic patients with minimal varices, no evidence of clinical bleeding and with an appropriate clinical indication.

Infact, the safety of prophylactic or therapeutic anticoagulation in carefully selected patients with cirrhosis without the presence of high-risk esophageal varices appears to be comparable to general medical patients. Also, in patients with esophageal varices, primary or secondary prophylaxis with either endoscopic variceal ligation or use of non-selective beta-blockers is suggested prior to initiation of anticoagulation and after discussing about the risks and benefits of anticoagulation with the patients. Periodical screening endoscopy should be considered to assess varices and the risk of bleeding in these patients. Various classes of heparin are currently available for prophylactic or therapeutic purposes including UFH, LMWH and fondaparinux. LMWH seems to be the treatment of choice for both the prevention and treatment of VTE in cirrhotic patients. UFH is an alternative in cirrhotic patients for shorter-term use and in cases of severe renal dysfunction and/or hemodynamic instability. The possible role for the new antithrombotic drugs with direct action on factor Xa or thrombin has yet to be evaluated.

Clinical tools to evaluate the risk of VTE in cirrhotic patient population also need to be developed to target the cirrhotic patient who will benefit most from the anticoagulation. Bogari $\mathrm{H}$ et al. [18] conducted a retrospective cohort study to evaluate the utilization of the Padua Predictor Score (PPS) as a risk-stratification tool for the development of VTE in 163 patients with liver cirrhosis [18,19].
They categorized the patients into two groups based on whether they developed a VTE (11\%) or not (89\%). Patients were further risk stratified into high-risk (score $\geq 4$ ) and low-risk (score <4) using the PPS. Patients in the VTE group had significantly greater mean PPS than in the non-VTE group $(5.8 \pm 2.0$ versus $3.0 \pm 2.1$, respectively; $\mathrm{p}<0.001)$. Also, High-risk patients were more likely to have VTE (OR 12.7, 95\% CI 2.8 to 57.4, p=0.001) as compared to low risk. Based on their findings, Bogari $\mathrm{H}$ et al. [18] concluded that PPS is an effective risk assessment tool for development of VTE in patients hospitalized with chronic liver disease. Various other factors like low serum albumin and decompensated liver cirrhosis have also shown to be associated with increased risk of VTE in patients with liver cirrhosis. In two retrospective studies conducted by Northup et al. [2], Garcia Fuster et al. [3] low serum albumin was found to be associated with increased risk of developing VTE independent from elevated INR or low platelet count [2,3]. Certain other factors like INR $>2$ and low platelet count may lower the risk of developing VTE. It will be prudent to develop tools including such factors to help accurately characterizing cirrhotic patients at higher risk of developing VTE. This will help provide clinicians with more confident to start anticoagulation in cirrhotic patients at high risk of bleeding secondary to esophageal varices.

Further studies are needed to determine the utility of novel methods to monitor the anticoagulation therapy in cirrhotic patients. LMWH is conventionally monitored by anti-Xa assay, while UFH can be monitored by activated PTT (aPTT) assay in the general population. However, monitoring of LMWH anticoagulation with anti-Xa levels for dose adjustment is challenging in cirrhotic patients since the levels of anti-Xa factor have been found to be lower in cirrhotic patients compared to normal controls after administration of prophylactic or therapeutic dose of LMWH [13]. For example, Bechmann et al. [13] studied the pharmacokinetics of LMWH in patients with cirrhosis and confirmed that standard doses of enoxaparin failed to achieve target anti Xa levels recommended for prophylactic or therapeutic use against VTE [13]. However, in vitro studies evaluating the effect of LMWH on thrombin generation found that despite reduced antithrombin and anti-Xa activity levels, cirrhotic patients had an increased response to LMWH [20]. They concluded that the likely explanation of low anti-Xa levels could be a laboratory artifact while the efficacy of LMWH is preserved [20]. Thus, targeting its levels for the prophylaxis and treatment of VTE in noncirrhotic patients might underestimate the true degree of anticoagulation which could lead to incorrect dosing and subsequent increase morbidity and mortality secondary to bleeding. The role of thrombin generation in monitoring the response to LMWH in cirrhotic patients has been investigated by Lisman T et al. [21] and they found it to be a useful tool and an alternative to anti-Xa levels [21]. On the other hand, UFH is monitored with aPTT in general population. But again, aPTT levels are also known to be prolonged in patients with liver cirrhosis thus making it difficult to predict the dose accuracy of UFH in this patient population. 
In the end, the treatment and prophylaxis for VTE should be utilized in patients with liver cirrhosis who are at low risk of bleeding i.e. does not have esophageal varices. Moreover, randomized prospective studies needs to be performed to confirm the efficacy of VTE prophylaxis in cirrhotic patients, to determine the safety of therapeutic anticoagulation against VTE in cirrhotic patients, to figure out accurate markers for monitoring the use anticoagulation in cirrhotic patients, and to better understand the role of coumadin and newer oral anticoagulants in VTE prophylaxis and treatment in cirrhotic patients.

\section{References}

1. Sharara AI, Rockey DC (2001) Gastroesophageal variceal hemorrhage. N Engl J Med 345(9): 669-681.

2. Northup PG, Mc Mahon MM, Ruhl AP, Altschuler SE, Volk-Bednarz A, et al. (2006) Coagulopathy does not fully protect hospitalized cirrhosis patients from peripheral venous thromboembolism. Am J Gastroenterol 101(7): 1524-1528.

3. Garcia Fuster MJ, Abdilla N, Fabiá MJ, Fernández C, Oliver V, et al. (2008) Venous thromboembolism and liver cirrhosis. Rev Esp Enferm 100(5): 259-262.

4. Dabbagh O, Oza A, Prakash S, Sunna R, Saettele TM (2010) Coagulopathy does not protect against venous thromboembolism in hospitalized patients with chronic liver disease. Chest 137(5): 1145-1149.

5. Anthony Lizarraga W, Dalia S, Reinert SE, Schiffman FJ (2010) Venous thrombosis in patients with chronic liver disease. Blood Coagul Fibrinolysis 21(5): 431-435.

6. Wu H, Nguyen GC (2010) Liver cirrhosis is associated with venous thromboembolism among hospitalized patients in a nationwide US study. Clin Gastroenterol Hepatol 8(9): 800-805.

7. Saleh T, Matta F, Alali F, Stein PD (2011) Venous thromboembolism with chronic liver disease. Am J Med 124(1): 64-68.

8. Aldawood A, Arabi Y, Aljumah A, Alsaadi A, Rishu A, et al. (2011) The incidence of venous thromboembolism and practice of deep venous thrombosis prophylaxis in hospitalized cirrhotic patients. Thromb J 9(1): 1 .

9. Ali M, Ananthakrishnan AN, Mc Ginley EL, Saeian K ( 2011) Deep vein thrombosis and pulmonary embolism in hospitalized patients with cirrhosis: a nationwide analysis. Dig Dis Sci 56(7): 2152-2159.
10. Gulley D, Teal E, Suvannasankha A, Chalasani N, Liangpunsakul S (2008) Deep vein thrombosis and pulmonary embolism in cirrhosis patients. Dig Dis Sci 53(11): 3012-3017.

11. Intagliata NM, Henry ZH, Shah N, Lisman T, Caldwell SH, et al. (2014) Prophylactic anticoagulation for venous thromboembolism in hospitalized cirrhosis patients is not associated with high rates of gastrointestinal bleeding. Liver Int 34(1): 26-32.

12. Vivarelli M, Zanello M, Zanfi C, Cucchetti A, Ravaioli, et al. (2010) Prophylaxis for venous thromboembolism after resection of hepatocellular carcinoma on cirrhosis: is it necessary? World J Gastroenterol 16(17): 2146-2150.

13. Bechmann LP, Sichau M, Wichert M, Gerken G, Kröger K, et al. (2011) Low-molecular-weight heparin in patients with advanced cirrhosis. Liver Int 31(1): 75-82.

14. Smith CB, Hurdle AC, Kemp LO, Sands C, Twilla JD (2013) Evaluation of venous thromboembolism prophylaxis in patients with chronic liver disease. J Hosp Med 8(10): 569-573.

15. Barclay SM, Jeffres MN, Nguyen K, Nguyen T (2013) Evaluation of pharmacologic prophylaxis for venous thromboembolism in patients with chronic liver disease. Pharmacotherapy 33(4): 375-382.

16. Reichert JA, Hlavinka PF, Stolzfus JC (2014) Risk of hemorrhage in patients with chronic liver disease and coagulopathy receiving pharmacologic venous thromboembolism prophylaxis. Pharmacotherapy 34(10): 1043-1049.

17. Sharara AI, Rockey DC (2001) Gastroesophageal variceal hemorrhage. N Engl J Med 345(9): 669-681.

18. Bogari H, Patanwala AE, Cosgrove R, Katz M (2014) Risk-assessment and pharmacological prophylaxis of venous thromboembolism in hospitalized patients with chronic liver disease. Thromb Res 134(6): 1220-1223.

19. Barbar S, Noventa F, Rossetto V, Ferrari A, Brandolin B, et al. (2010) A risk assessment model for the identification of hospitalized medical patients at risk for venous thromboembolism: the Padua Prediction Score. J Thromb Haemost 8(11): 2450-2457.

20. Senzolo M, Rodriguez-Castro KI, Rossetto V, Radu C, Gavasso S, et al. (2012) Increased anticoagulant response to low-molecular-weight heparin in plasma from patients with advanced cirrhosis. J Thromb Haemost 10(9): 1823-1829.

21. Lisman T, Porte RJ (2011) Towards a rational use of low-molecularweight heparin in patients with cirrhosis. Liver Int 31(7): 1063.

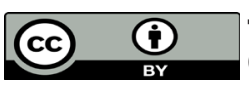

This work is licensed under Creative Commons Attribution 4.0 License

To Submit Your Article Click Here: Submit Article

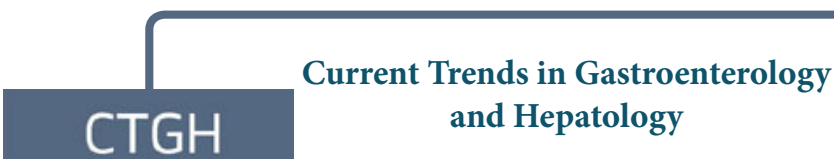

Assets of Publishing with us

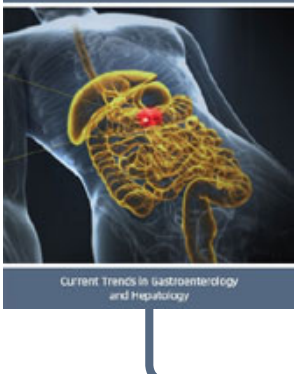

- Global archiving of articles

- Immediate, unrestricted online access

- Rigorous Peer Review Process

- Authors Retain Copyrights

- Unique DOI for all articles 